\title{
Investigation of the BOLD-Based MRI Signal Time Course During Short Breath-Hold Periods for Estimation of the Cerebrovascular Reactivity
}

\author{
Leonie Zerweck $^{1}$ (D) $\cdot$ Till-Karsten Hauser $^{1}$ (D) Constantin Roder ${ }^{2}$ (D) Uwe Klose $^{1}$ (D)
}

Accepted: 28 July 2020 / Published online: 10 August 2020

(C) The Author(s) 2020

\begin{abstract}
For the prognosis of stroke, patients with moyamoya disease (MMD) require the estimation of remaining cerebrovascular reactivity. For this purpose, $\mathrm{CO}_{2}$-triggered $\mathrm{BOLD}$ fMRI by use of short breath-hold periods seems to be a highly available alternative to nuclear medicine methods. Too long breath-hold periods are difficult to perform, too short breath-hold periods do not lead to sufficient BOLD signal changes. We aimed to investigate the required minimum breath-hold duration to detect distinct BOLD signals in the tissue of healthy subjects to find out how long the minimum breath-hold duration in clinical diagnostics of MMD should be. A prospective study was performed. Fourteen healthy subjects underwent fMRI during end-expiration breathhold periods of different duration (3, 6, 9, and $12 \mathrm{~s}$ ). Additionally, we compared the influence of paced and self-paced breathing altering the breath-hold periods. Data of a patient with MMD was evaluated to investigate whether the tested procedure is suitable for clinical use. Significant global BOLD signal increases were detected after breath-hold periods of 6, 9, and $12 \mathrm{~s}$. The signals were significantly higher after breath-hold periods of $9 \mathrm{~s}$ than after $6 \mathrm{~s}$, while not when the duration was extended from 9 to $12 \mathrm{~s}$. Furthermore, we found additional BOLD signal changes before the expected signal increases, which could be avoided by paced respiratory instructions. This investigation indicates that end-expiration breath-hold period of at least $9 \mathrm{~s}$ might be used to measure the cerebrovascular reactivity. This time period resulted in distinct BOLD signal changes and could be performed easily.
\end{abstract}

Keywords Functional magnetic resonance imaging - Cerebrovascular reactivity · Moyamoya disease Hypercapnia Breath-holding

\section{Introduction}

Moyamoya disease (MMD) is a cerebrovascular disease that leads to progressive stenosis of terminal parts of the internal carotid arteries (ICA) and the circulus of Willis. The reactively formed collateral vessels serving to maintain the cerebral blood flow (CBF) remind on angiograms of MMD patients

This article is part of the Topical Collection on Medicine

Leonie Zerweck

leonie.zerweck@med.uni-tuebingen.de

1 Department of Diagnostic and Interventional Neuroradiology, University Hospital Tuebingen, Eberhard Karls University Tuebingen, Hoppe-Seyler-Str. 3, 72076 Tuebingen, Germany

2 Department of Neurosurgery, University Hospital Tuebingen, Eberhard Karls University Tuebingen, Hoppe-Seyler-Str. 3, 72076 Tuebingen, Germany of "puff of smoke" (Japanese: "moyamoya") and therefore gave the disease its name. The most frequent symptoms of patients with MMD are ischemic or hemorrhagic strokes and transient ischemic attacks (TIA) $[1,2]$. A causal therapy option does not exist; the treatment is merely symptomatic, for example, bypass surgery between terminal vessels of the internal carotid artery (ICA) and external carotid artery system [3]. Angiography currently receives the most attention in diagnostics $[1,2]$. However, the indication for surgical therapy is based on evidence of reduced cerebrovascular reactivity (CVR) through functional perfusion imaging [4]. Nuclear medicine imaging techniques are much used to investigate the cerebrovascular reactivity, but are limited available due to the requirement of short-lived radiopharmaceutical tracers and relatively expensive [5]. Functional magnetic resonance imaging (fMRI) technique can also be employed in clinical routine to determine remaining cerebrovascular reactivity at patients with MMD using the exogenous vasoactive stimulus acetazolamide (ACT) [6] or hypercapnic challenges such as 
inhalation of $\mathrm{CO}_{2}$-enriched gas [7-10] or breath-holding [11]. Hypercapnia is known to induce cerebral vasodilatation in healthy brain tissue and increased cerebral blood flow (CBF) [6]. The resulting altered ratio of paramagnetic deoxyhemoglobin to diamagnetic oxy-hemoglobin in venules leads to blood-oxygen-level-dependent (BOLD) signal increase due to different magnetic susceptibility [12]. In vascular territories with insufficient CVR, reduced increase in perfusion during hypercapnic stimulation is expected, as the vessels are chronically in a state of maximum dilatation to maintain cerebral blood flow. Therefore, in patients with MMD, the BOLD signal increase is not supposed to be observed in the pathological vascular territories. In the case of a vascular steal phenomenon, the blood flow in the affected area is even reduced at the expense of adjacent tissue and a signal drop can be measured $[10,13]$. Breath-hold technique can be a wellpracticable method to achieve hypercapnia. It is noninvasive and does not require any additional equipment $[14$, 15], but leads to similar BOLD signal changes as the inhalation of $\mathrm{CO}_{2}$-enriched gas mixtures [15].

A recent retrospective study showed that in patients with MMD, the hypercapnia-triggered fMRI by use of short breathhold periods leads to comparable results to the more complex and expensive positron emission tomography (PET) with acetazolamide challenge [11]. An open question in this investigation was the necessary duration of the breath-hold periods for clinical use. Therefore, our aim was to investigate prospectively the required minimum breath-hold duration to detect distinct BOLD signal increases in healthy brain tissue. This time period might be used in clinical routine to distinguish pathological vascular territories where no signal increase can be measured. A compromise between reliable change in the BOLD signal intensity and the patients' tolerance limit is necessary. Long breath-hold periods are associated with difficulties in carrying out the tasks, particularly for patients with limited state of health; too short breath-hold periods do not provide sufficient BOLD signals. In the implementation of breath-hold tasks, a distinction is made between breath-hold (BH) following inspiration (end-inspiration) $[12,16-18]$ and BH following expiration (end-expiration) [11, 12, 19-23]. BH periods after expiration are supposed to lead to earlier and higher signal increases [21]. Furthermore, after endexpiration $\mathrm{BH}$ periods, less signal variability is expected than after end-inspiration $\mathrm{BH}$ periods, where differing inspiration depth might occur [24], attributable to the fact that lungs, diaphragm, and chest are in an equilibrium state after expiration [23]. To abbreviate the time period where subjects are required to stop breathing and to ensure high reproducibility, we decided to investigate end-expiration $\mathrm{BH}$ periods.

Additionally, we performed a task with a paced breathing rhythm to prevent unexpected BOLD signal changes due to irregularities in the respiration of the subjects and compared the signal time courses during paced and self-paced breathing.

\section{Methods}

A prospective functional MRI study of healthy subjects and a retrospective comparison with the data of one patient with moyamoya disease were performed.

Subjects Fourteen healthy subjects (10 female, 4 male, mean age: 25 , range: $21-40$ ) without known cerebrovascular disease were included in this study. Written informed consent was obtained from each participant after the design of study was explained. The study protocol was approved by the local Ethics Committee.

Additionally, data of one patient with unilateral moyamoya disease was evaluated. The data was acquired during a routine MRI scan to evaluate cerebral reserve capacity before extraintracranial bypass surgery. The patient presented to our hospital after multiple small ischemias were detected during a workup for recurrent hypesthesia of the left arm. The patient consented to the evaluation and publication of the acquired data.

MRI Data Acquisition Subjects' experiments were performed on a 3-T scanner (Siemens MAGNETOM Trio Tim syngo MR B17, Erlangen, Germany). A standard 20-channel head coil was used for imaging. The data was measured by means of a $\mathrm{T} 2 *$-weighted echo-planar imaging sequence with the following parameters: TR $3000 \mathrm{~ms}$; TE $36 \mathrm{~ms}$; flip angle $90^{\circ} ; 3 \mathrm{~mm}$ slice thickness, 34 slices, FOV $245 \mathrm{~mm}$, matrix size $96 \times 96$; TA 6:53, resolution: $2.6 \times 2.6 \times 3.0 \mathrm{~mm}, 135$ measurements.

Physiological Monitoring During the examination, subjects were positioned supine on the scanner table.

The respiratory movements of 11 subjects were measured with a pneumatic abdominal belt. For technical reasons, the respiratory movement of 3 subjects could not be measured.

Experimental Paradigm Each subject performed 4-8 tasks with different respiratory paradigms during separate functional scans. Each scan consisted of five repetitive cycles with a duration of $69 \mathrm{~s}$. Each cycle included two blocks: the breath-hold block and the breathing block. As shown in Fig. 1, each cycle ended with the breath-hold block. After the last period, an additional time period with a duration of $60 \mathrm{~s}$ was performed to detect time-delayed signal changes. During the breath-hold blocks, subjects were instructed to hold their breath for 3, 6, 9 or $12 \mathrm{~s}$ (BH time) according to the different conditions. During the breathing blocks, subjects were asked to maintain natural breathing in their own rhythm for $66,63,60$, or $57 \mathrm{~s}$ (BN time), depending on the $\mathrm{BH}$ time $(3,6,9$, or $12 \mathrm{~s})$. Breathing blocks included a preparation stage during which subjects were asked to breathe in $(2 \mathrm{~s})$ and breathe out $(3 \mathrm{~s})$ to initiate the breath-hold period after expiration. Respiratory instructions were given verbally. 
Fig. 1 Bottom: BOLD-functional MRI activation paradigm: 5 repetitive cycles including $\mathrm{BN}$ time (yellow) and $\mathrm{BH}$ time (red).

The 5 cycles of $69 \mathrm{~s}(23$

measurements) each were followed by $60 \mathrm{~s}(20$ measurements) of regular breathing. Top: detailed view: one cycle consists of the instructions "breathe normally," "breathe in" (i), "breathe out" (o), and "do not breathe"

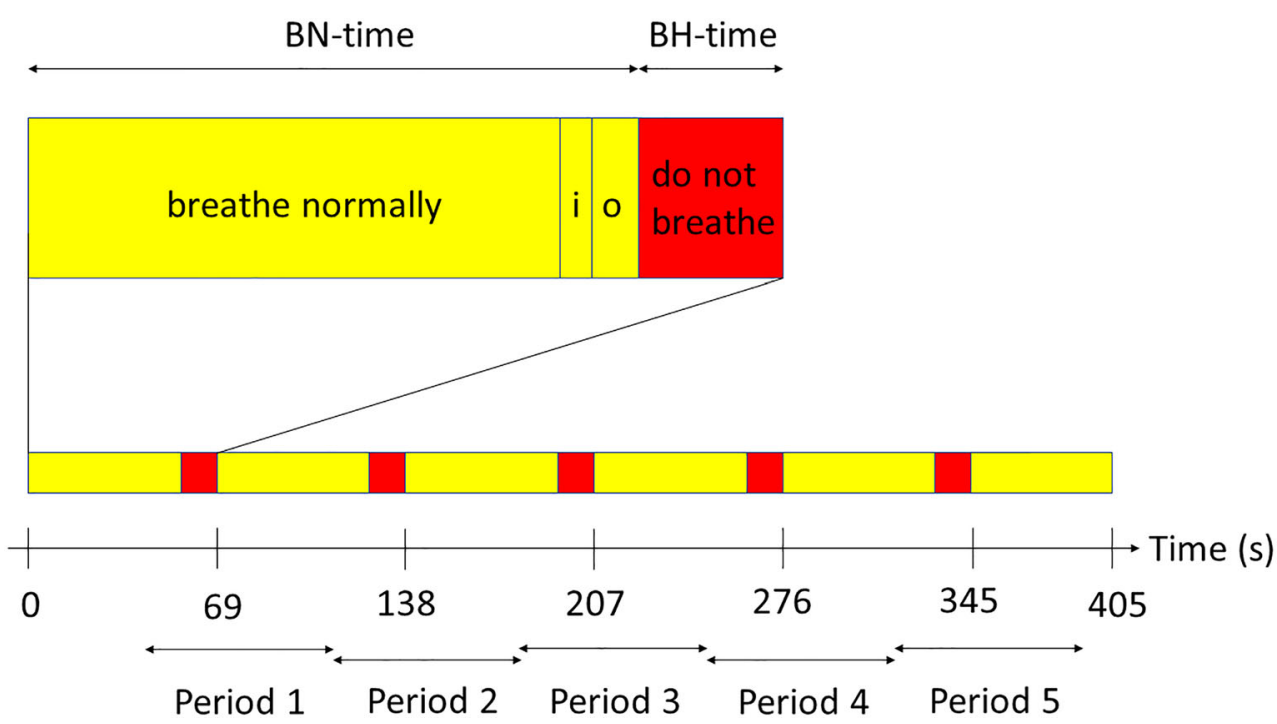

Subjects were instructed to avoid deep inspiration before the $\mathrm{BH}$ period.

The 12 -s BH task was performed by 9 subjects, the 9 -s BH task by 14 subjects, the 6 -s BH task by 10 subjects, and the $3-\mathrm{s}$ BH task by 9 subjects. The reason for the different numbers of subjects performing the task was limited measuring time. To assess the intra-subjects' variability, one of the 14 subjects performed the 9-s BH task 6 times on two different days (1 measurement on day one, 5 measurements 8 months later). In total, the 9-s BH task with altering self-paced breathing was therefore performed 19 times.

To analyze the signal time course without signal changes due to irregularity in the subjects' natural breathing rhythm, a 9-s paced breathing task was performed additionally by 8 subjects. Within this task, subjects were only allowed to breathe when the instructions "breathe in" and "breathe out" were given during BN time. The respiratory frequency was chosen at 12 breaths/min, with 2 -s inspiration and $3-\mathrm{s}$ expiration.

MRI Data Processing and Analysis Image were preprocessed using Statistical Parameter Mapping (SPM12) (https://www. fil.ion.ucl.ac.uk/spm/) running on MATLAB (R2018b (The MathWorks, Inc., Natick, Massachusetts; http://www. mathworks.com)). Images were converted to Analyze format in NIfTI (Neuroimaging Informatics Technology Initiative) and then slice time corrected to compensate the different points in time of image acquisition, realigned to correct subjects' head movement, normalized to standard MNI (Montreal Neurological Institute) space, and spatially smoothed by a Gaussian kernel of $8 \mathrm{~mm}$ full width at half maximum.

All further processing of the data was performed using a script programmed in MATLAB (R2018b (The MathWorks,
Inc., Natick, Massachusetts; http://www.mathworks.com)). Four templates (territories of anterior cerebral artery (ACA), middle cerebral artery (MCA), posterior cerebral artery (PCA) , and cerebellar vascular territories) based on the arterial transit time based flow territories [25] were utilized. The results were averaged and presented as a common signal course. The percentage of signal change was calculated from the raw data relative to baseline level, which was seen $42-60 \mathrm{~s}$ after the beginning of the breath-hold period. The signal time course was averaged over 5 time periods. The vertical yellow lines in Fig. 2 indicate the 5 time periods over which averaging was done. In order to map the time-delayed signal change, these 5 periods were time-shifted to the 5 cycles of the instructions. Figure $2 \mathrm{~b}$ shows an exemplary resulting signal course after averaging over the 5 periods. In order to compare the effects of different respiratory paradigms, results of identical paradigms were grouped together and averaged over all subjects.

As seen in Table 1, the maximum signal change, standard deviation of the maximum signal change, and time to peak (TTP), that is defined as the time from the beginning of the $\mathrm{BH}$ period to maximum signal increase, were calculated. A single sample $t$ test was applied to assess the maximum BOLD signal changes of all measurements of each task at the expected TTP (12-s BH task: $24 \mathrm{~s}, 9-\mathrm{s}$ BH task: $21 \mathrm{~s}, 6$-s BH task: $18 \mathrm{~s}$, see Table 1). The 3-s BH task did not show a signal increase analogous to the results of longer BH duration. Therefore, $15 \mathrm{~s}$ was defined as TTP according to the findings of the other results. A paired sample $t$ test was performed to test the differences between the BOLD signal changes after breath-hold periods of different durations at the expected TTP comparing the results of all subjects who performed all tasks. $p<0.05$ was considered statistically significant. 


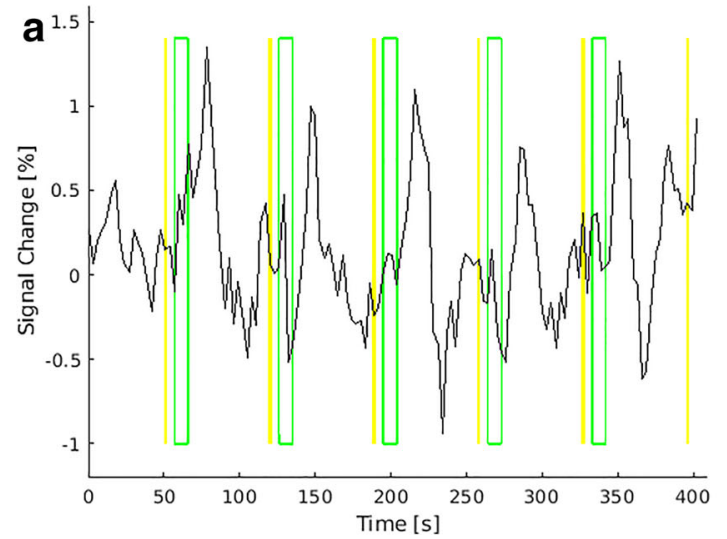

Fig. 2 a Signal time course of one exemplary subject performing the 9-s breath-hold task. The phases in which the subject did not breathe are marked by green rectangles. The vertical yellow lines symbolize the start and end of the five periods over which averaging was done. $\mathbf{b}$

To evaluate the reproducibility, the intra-subject's variability was estimated by calculating of the coefficient of variation (CV) (standard deviation/mean) of the maximum signal increase and the TTP among the 6 measurements of one subject. We calculated the maximum signal changes during the expected time period (TTP $\pm 3 \mathrm{~s}$ ) of each measurement of all $\mathrm{BH}$ paradigms in order to compare the reproducibility of signal increases between the $\mathrm{BH}$ paradigms of different $\mathrm{BH}$ duration.

To investigate differences between the signal time courses during the paced and the self-paced breathing task, the amplitude of the maximum signal change and the TTP of all measurements of both tasks were compared by means of a twosample $t$ test. In addition, to compare the inter-trial variability during the paced and the self-paced breathing task, the coefficient of variation between all 5 signal increases of one single measurement was calculated. The signal increases were defined as the difference of the lowest BOLD signal value after the beginning of the $\mathrm{BH}$ period and the following highest signal value. The coefficients of variation of all subjects performing paced and self-paced breathing tasks were compared by use of a paired sample $t$ test.

Table 1 Overview of the measurements of different BH time

\begin{tabular}{lllll}
\hline Breath-hold time & $12 \mathrm{~s}$ & $9 \mathrm{~s}$ & $6 \mathrm{~s}$ & $3 \mathrm{~s}$ \\
\hline Percent signal change [\%] & $0.9^{*}$ & $0.8^{*}$ & $0.3^{*}$ & na \\
Standard deviation [\%] & 0.3 & 0.1 & 0.2 & na \\
TTP (time to peak) [s] & 24 & 21 & 18 & na \\
Number of evaluated measurements & 8 & 13 & 9 & 9 \\
\hline
\end{tabular}

na not applicable due to missing peak

An asterisk (*) marks significant values at $p<0.05$

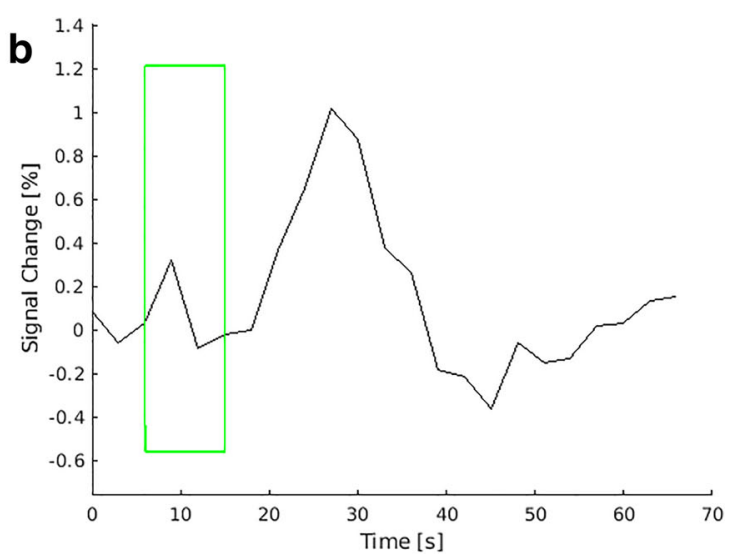

Signal time course of the exemplary subject averaged over the five periods. The percentage BOLD signal change is shown as percentage increase compared with baseline level. The green rectangle marks the $\mathrm{BH}$ period

Activation maps of one exemplary subject and one patient with MMD during the identical 9-s BH task were calculated by voxel-wise integration of the signal time course during the time period of the expected maximum signal increase (TTP $\pm 6 \mathrm{~s}$ ). The integral values of a time period, in which we did not expect any signal changes, but baseline signal (27-39 s after the end of the BH period), were subtracted. The maps show the integral values of each voxel relative to the mean integral values of the cerebellum. The cerebellum was selected as region of reference, because in patients with MMD, the vessels supplying the cerebellum usually do not reveal MMD-typical stenosis.

\section{Results}

\section{Evaluation of Respiratory Movement}

No subject reported any difficulties executing the respiratory instructions. Evaluation of the respiratory movement monitoring revealed that all subjects except one respected the $\mathrm{BH}$ periods of 3 to $12 \mathrm{~s}$, and that the $\mathrm{BH}$ periods were carried out in end-expiration. Because of irregularities in the subject's breathing rhythm and incorrect execution of the respiratory instructions, the results of this subject were not included in the evaluation.

However, it was noticeable by breath monitoring that some subjects carried out the inspiration immediately before the BH period in a deeper way. In some of these cases, the respiratory instruction "breathe in" fell into a period of their self-paced breathing rhythm inspiration. During the paced breathing task, a steady breathing rhythm with uniform respiratory rate and inhalation depth was observed. 


\section{Signal Changes After Variable BH Time}

As seen in Fig. 3, tasks of variable BH time (12 s, 9 s, $6 \mathrm{~s}$ ) led to similar shapes of signal time courses, although the maximum signal changes and the TTP differed. Significant BOLD signal increases could be detected after breath-hold periods of $12 \mathrm{~s}\left(p<10^{-4}\right), 9 \mathrm{~s}\left(p<10^{-8}\right)$, and $6 \mathrm{~s}\left(p<10^{-2}\right)$. The prolongation of the $\mathrm{BH}$ period was accompanied by an increase in the maximum BOLD signal. The 12-s and 9-s BH task resulted in maximum signal changes of $0.9 \%$ and $0.8 \%$, while the 6 -s BH period led to signal changes of only $0.3 \%$. During the $3-\mathrm{s}$ BH task, no significant BOLD signal increase comparable to the measurements with longer $\mathrm{BH}$ time could be observed. The results of the paired sample $t$ test revealed that the BOLD signal values after $\mathrm{BH}$ periods of $9 \mathrm{~s}$ were significantly higher than after BH periods of $6 \mathrm{~s}\left(p<10^{-2}\right)$. The differences between the BOLD signal values after BH periods of 9 and $12 \mathrm{~s}$ were not significant. The TTP of the maximum signal increase was later when the breath-hold duration was lengthened (12-s BH task: 24 s, 9-s BH task: $21 \mathrm{~s}, 6-\mathrm{s}$ BH task: $18 \mathrm{~s}$ ). Before the maximum signal increase, significant $\left(p<10^{-5}\right)$ initial signal peaks could be detected, achieving their maximum $(0.3 \%) 3 \mathrm{~s}$ after the start of the breath-hold periods. Table 1 gives an overview of the percentage signal change, standard deviation, TTP, and the number of test runs. As seen in Fig. 4, distinct signal increases during the expected time period were measured in all measurements after $\mathrm{BH}$ periods of 9 and $12 \mathrm{~s}$. After BH periods of 3 and $6 \mathrm{~s}$, the expected signal increase could not be reliably observed in all measurements. In some cases, only small or even negative signal changes during the expected time period were detected.

\section{Intra-Subjects' Reproducibility}

All 6 test runs of one subject, who performed the identical BH task on two different days, revealed distinct signal increases. Figure 5 shows the averaged signal time course of the 6 results of one subject, and the time course \pm the corresponding standard deviation of each measurement point. Significant BOLD signal increases $\left(p<10^{-3}\right)$ of $1.1 \%$ on average were detectable. The standard deviation at the time point of the maximum signal increase accounted for $0.1 \%$, and the coefficient of variation was 0.09 . The mean TTP was $21.5 \mathrm{~s}$. The standard deviation of TTP amounted to $1.2 \mathrm{~s}$ and the coefficient of variation was 0.06 .

\section{Paced Versus self-Paced Breathing}

Comparing the signal time course of the paced breathing task and the task with self-paced breathing (Fig. 6), no significant differences in the TTP (mean TTP in both tasks: $21 \mathrm{~s}$ ) and the maximum BOLD signal changes (paced breathing task: $0.9 \%$ versus self-paced breathing
Fig. 3 Signal time courses of different $\mathrm{BH}$ time averaged over the five periods and over all subjects. The first thick vertical black line symbolizes the beginning of the $\mathrm{BH}$ period of all measurements. The 4 colored rectangles represent the $\mathrm{BH}$ time of the different measurements. The graphs are presented in the corresponding color

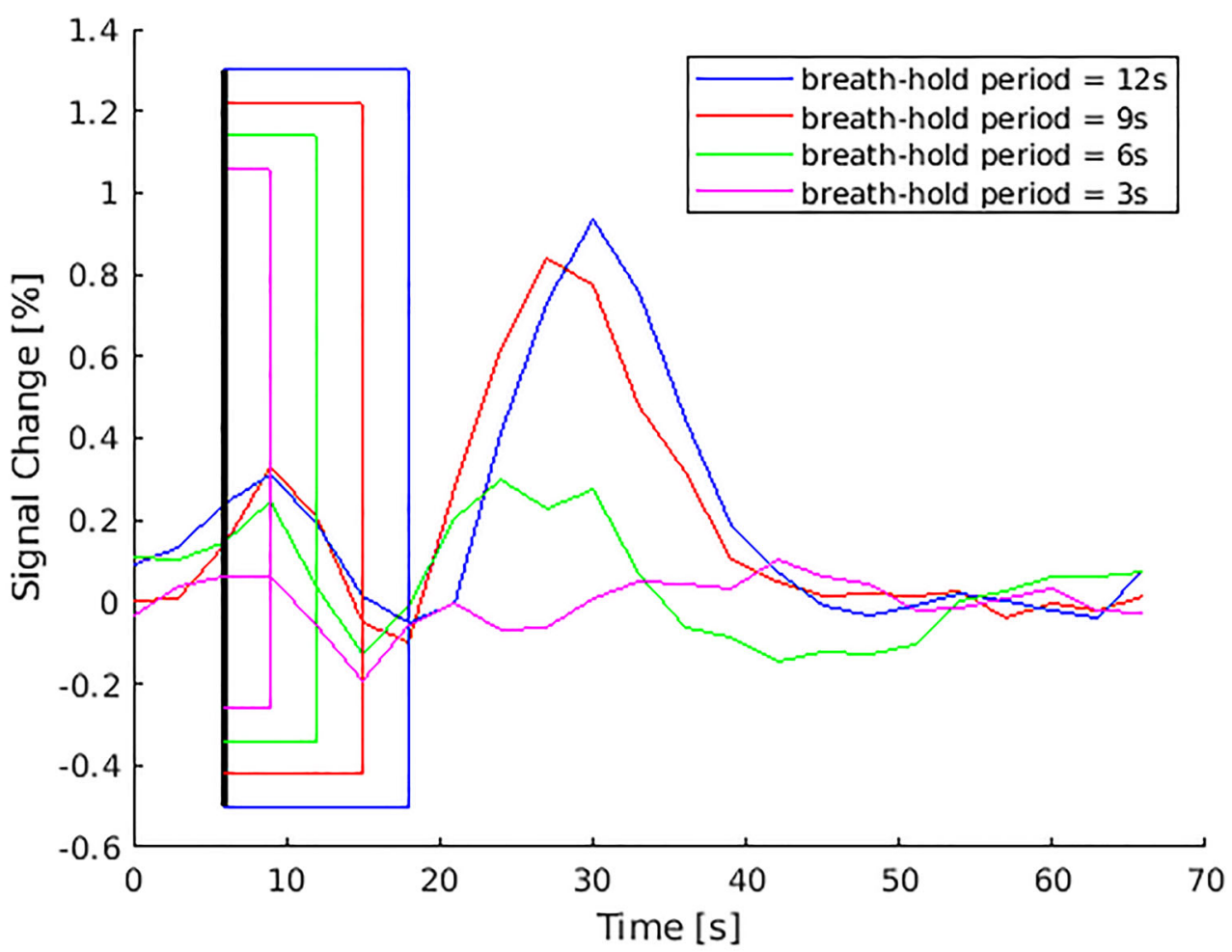


Fig. 4 Comparison of the maximum BOLD signal changes during the expected time period (TTP $\pm 3 \mathrm{~s}$ ) of all measurements of the BH tasks of different duration. The measurements are sorted in ascending order by amplitude of the maximum signal changes. BH periods of 9 and $12 \mathrm{~s}$ resulted in distinct signal increases during the expected time period in all measurements, while after $\mathrm{BH}$ periods of 3 and $6 \mathrm{~s}$, in some cases, only small or even negative signal changes were measured

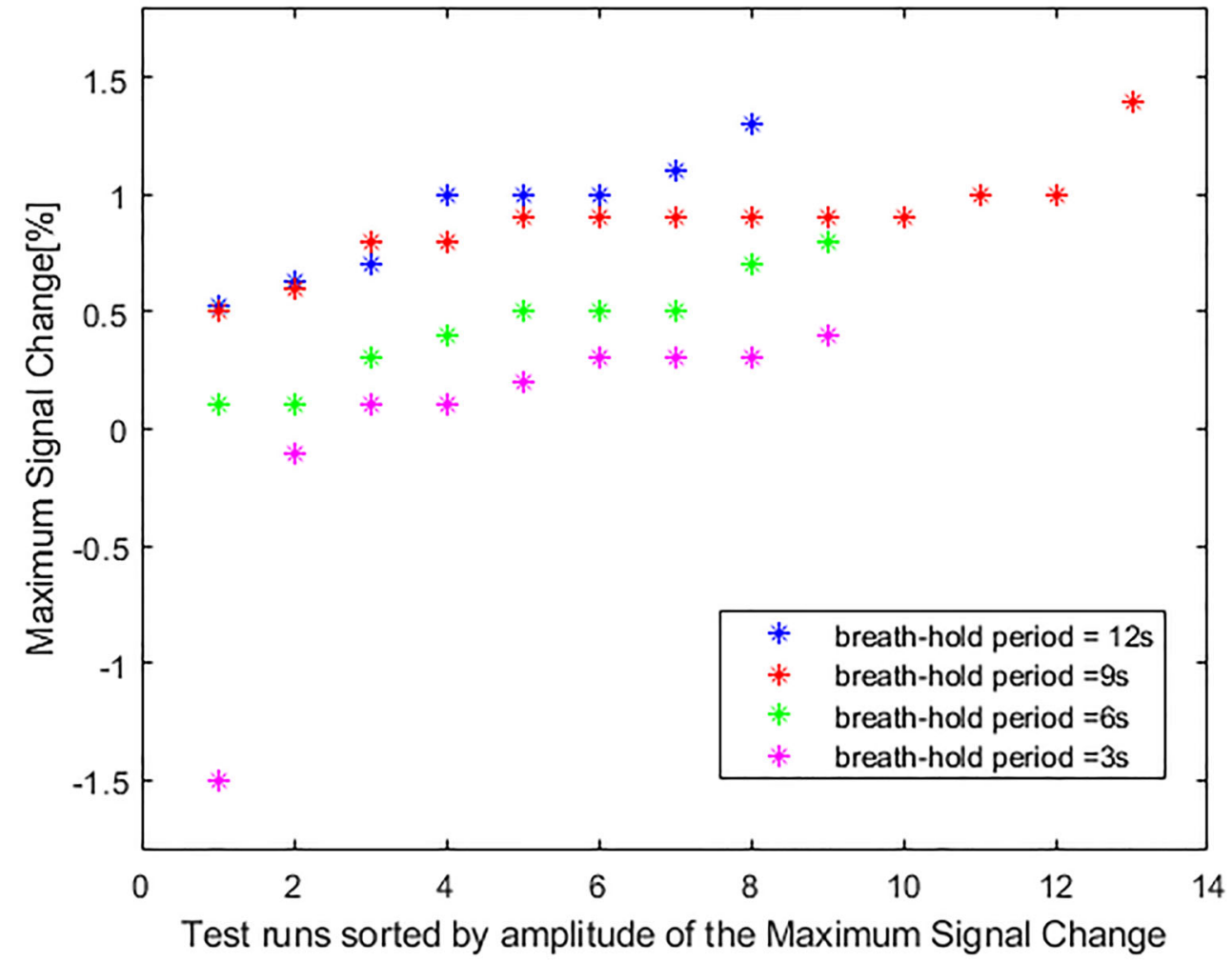

task: $0.8 \%$ ) were seen. As opposed to the self-paced breathing task, no significant additional initial peak (see asterisk (*) at Fig. 6) could be detected during the paced breathing task, whereas a stable baseline level before the expected main signal increase was observed. During the self-paced breathing task, the initial peak was observed in all measurements. By using paced respiratory instructions, the initial peak could be completely avoided in 7 of 8
Fig. 5 Signal time course of one subject performing 6 test runs of the 9-s BH task: the black line represents the mean signal time course averaged over all test runs and all 5 periods. The blue lines visualize the signal time course \pm the standard deviation at the corresponding time point

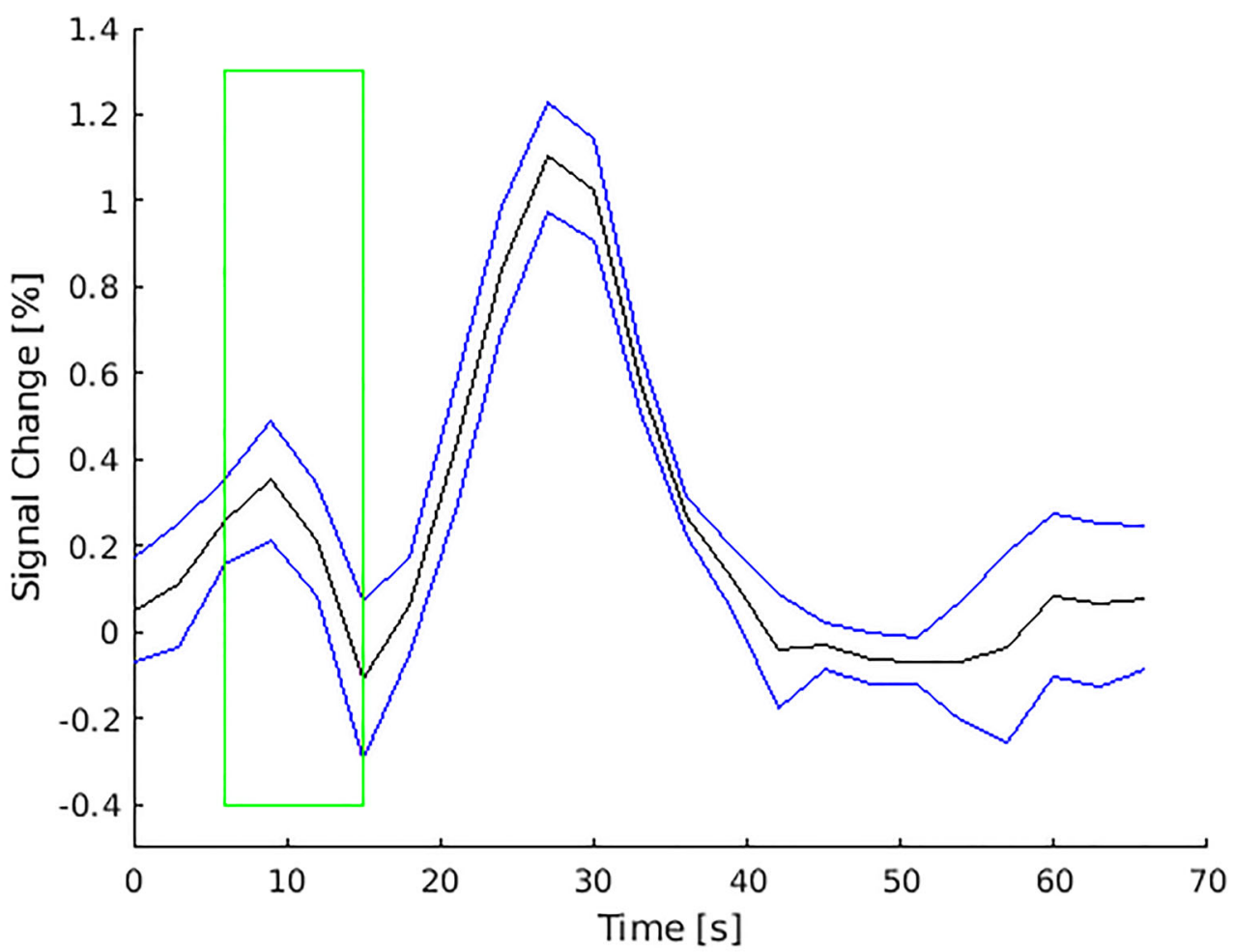


Fig. 6 Comparison of the mean signal time courses of the 9-s BH task during self-paced breathing and paced breathing of all subjects who performed the respective task. The green rectangle marks the $\mathrm{BH}$ period. The asterisk (*) marks the initial signal increase during the selfpaced breathing task which cannot be seen during the paced breathing task

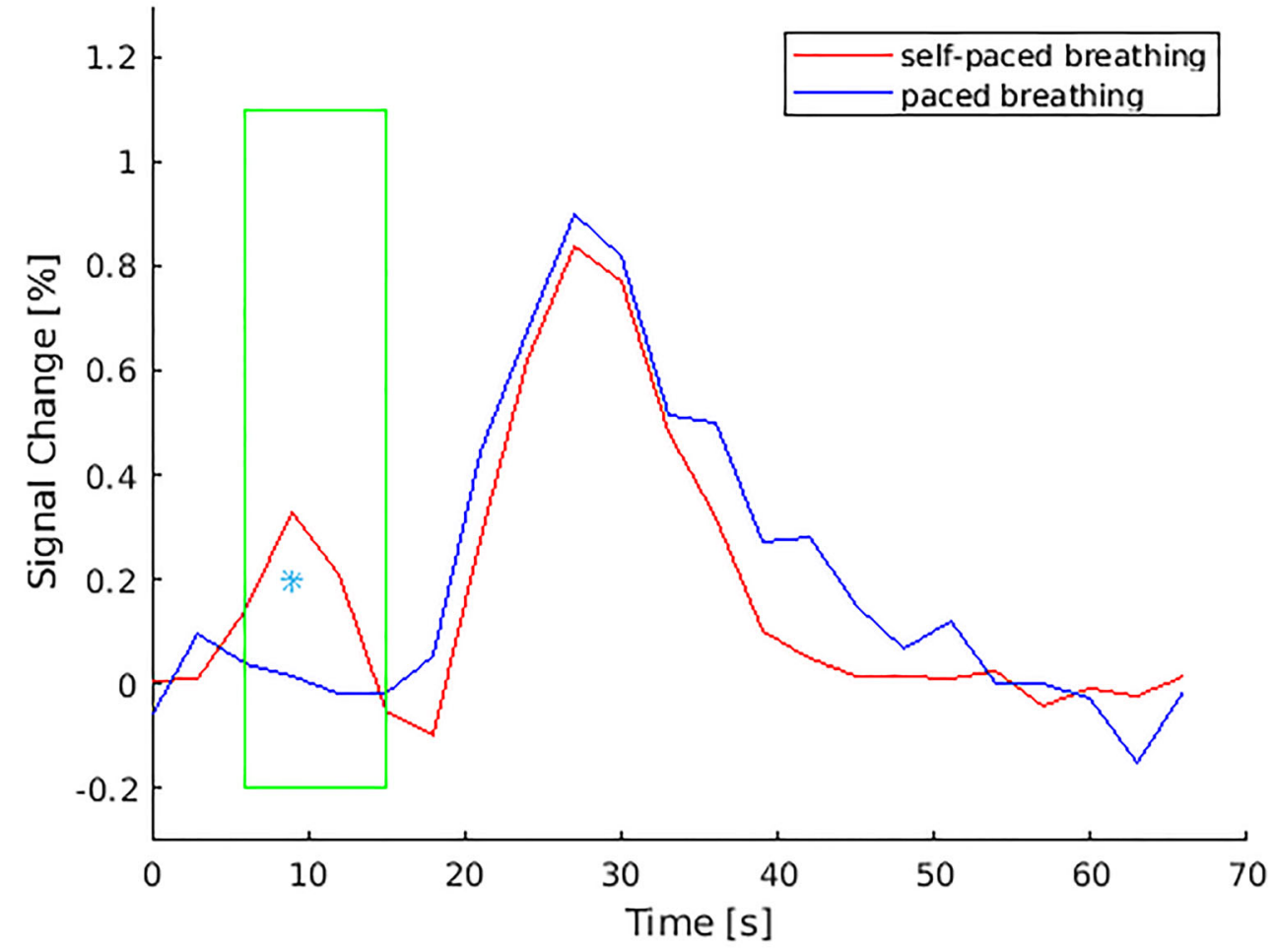

measurements. During the paced breathing task, the BOLD signal recovered slightly later to baseline level. The investigation of the inter-trial variability by comparing the coefficient of variation of the 5 signal maxima of one measurement during paced (0.24) and self-paced 9-s $\mathrm{BH}$ task (0.23) revealed no significant differences. One subject with irregular breathing rhythm showed a more stable baseline level after the signal increase during the paced breathing task than during the self-paced breathing task.

\section{Activated Regions}

As seen in Fig. 7a, the map of one healthy subject showed global symmetrical activation in the gray matter of the whole brain. Distinct BOLD signal increases were detectable in the vascular territories of the ACA, the MCA, the PCA, and the cerebellum, with no relevant differences between the right and left hemisphere (Fig. 8a). The map of the patient with MMD (Fig. 7b) revealed an asymmetric distribution pattern of activated brain regions. Compared with the reference region of the unaffected cerebellum, distinctly reduced activation was measured in some regions of gray matter. Particularly, the vascular territories of the right MCA, where multiple small ischemias were detected, revealed lower activation compared with the left hemisphere. The lower activation in the vascular territories of the right MCA was also evidenced by the reduced signal increase in this region, whereas the signal time course of the left MCA territory showed a nearly normal response curve (Fig. 8b).

\section{Discussion}

\section{Signal Changes After Variable BH Time}

One of the major findings of this study is that significant global BOLD responses can be detected after $\mathrm{BH}$ periods of 6 , 9, and $12 \mathrm{~s}$, whereas $\mathrm{BH}$ periods of $3 \mathrm{~s}$ do not induce significant global signal increases. Another important finding is that the BOLD signals after $\mathrm{BH}$ periods of $9 \mathrm{~s}$ were significantly higher than after $\mathrm{BH}$ periods of $6 \mathrm{~s}$, while the extension from 9 to $12 \mathrm{~s}$ revealed no significant additional signal increase. After $\mathrm{BH}$ periods of 9 and $12 \mathrm{~s}$, the expected signal increases were observed in all measurements, but shorter $\mathrm{BH}$ periods did not result in reliable signal increases. Therefore, in clinical diagnostics, $\mathrm{BH}$ periods of at least $9 \mathrm{~s}$ seem to be advisable. This assumption is also supported by the high intra-subjects' reproducibility after $\mathrm{BH}$ periods of $9 \mathrm{~s}$.

Long BH periods, especially after expiration, might be difficult to perform for sick and elderly patients. Particularly, patients with cardiopulmonary diseases may have problems performing long $\mathrm{BH}$ periods. As shorter $\mathrm{BH}$ periods are easier to execute and $\mathrm{BH}$ periods of 9 and $12 \mathrm{~s}$ lead to comparable results, $\mathrm{BH}$ periods of $9 \mathrm{~s}$ might be recommended [26, 27].

The suitability of the method to measure the cerebrovascular reactivity in clinical routine by use of short end-expiration 


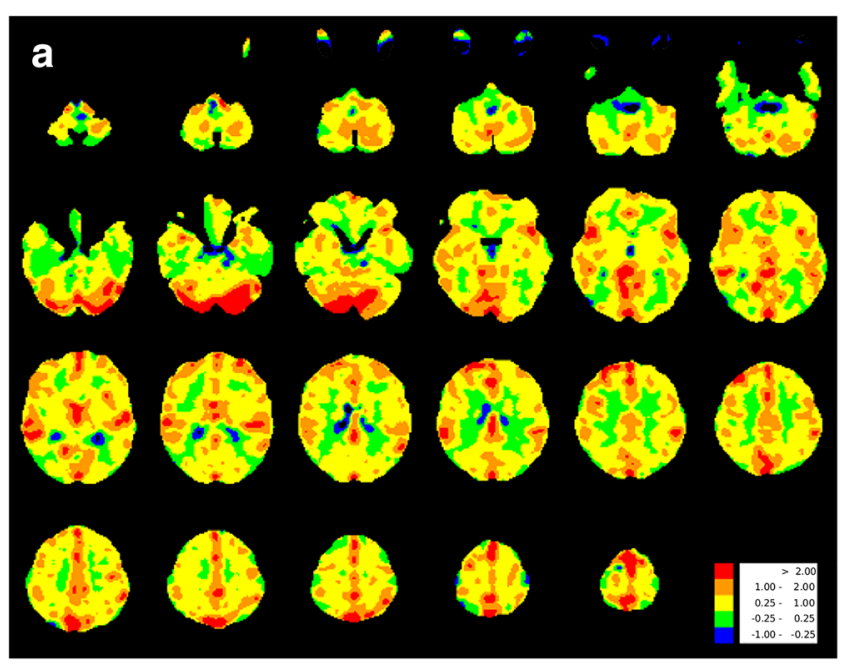

Fig. 7 Activation map of one exemplary healthy subject (a) and one patient with moyamoya disease (b) during the 9-s BH task calculated by voxel-wise integration of the signal time course. The color bar represents the integral values relative to the integral values of the

$\mathrm{BH}$ periods of $9 \mathrm{~s}$ was demonstrated exemplarily in one patient with MMD. In the evaluation of the BOLD signal time courses, as well as in the activation maps, reduced cerebrovascular reactivity was detected in the vascular territories of the affected vessel. The comparison of the maps of one healthy subject and a patient with MMD revealed that the reduced cerebrovascular reactivity of territories of moyamoya-typical stenosed vessels could be visualized by use of the 9-s breathhold paradigm.

This is consistent with the analysis of Hauser et al. in which breath-hold periods of $9 \mathrm{~s}$ resulted in comparable estimation of

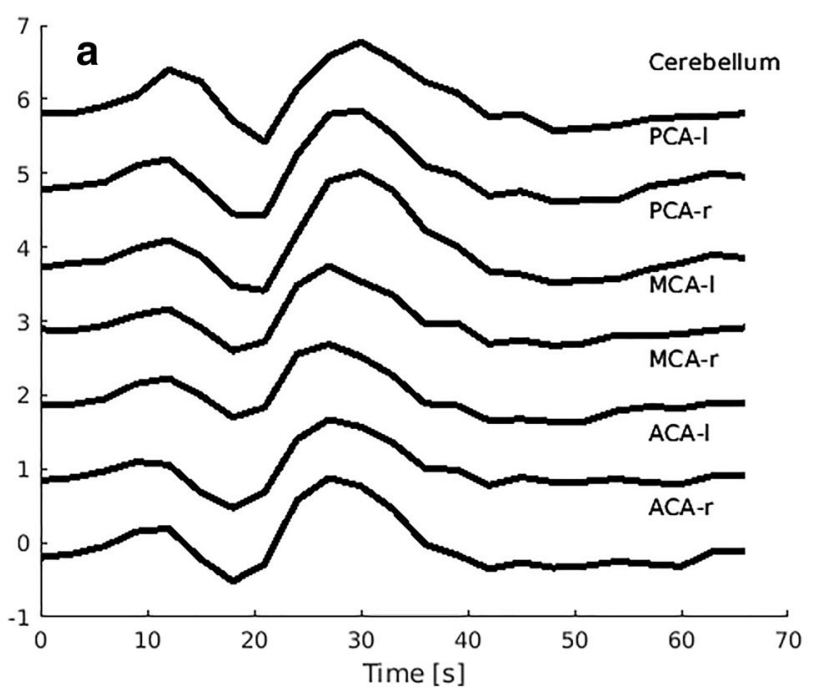

Fig. 8 BOLD signal time course of different regions (cerebellum, left posterior cerebral artery (PCA-1) right posterior cerebral artery (PCA-r), left middle cerebral artery (MCA-l), right middle cerebral artery (MCAr), left anterior cerebral artery (ACA-1), right anterior cerebral artery (ACA-r)) averaged over the 5 periods in one healthy subject (a) and

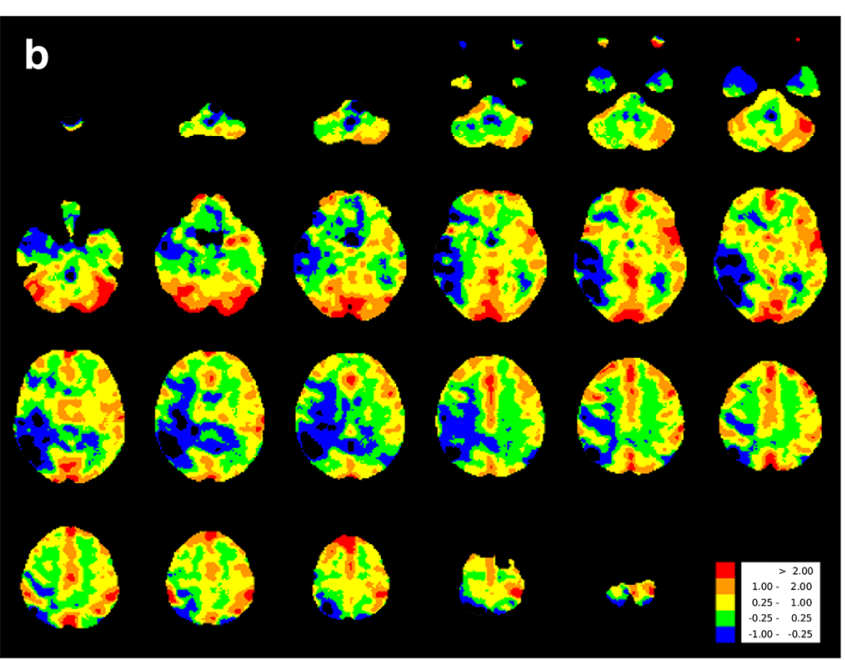

cerebellum. Breath-hold fMRI of the patient with moyamoya disease showed severe reduction of cerebral reserve capacity in the territory of the right middle cerebral artery (MCA) caused by an occlusion of the right MCA which was only supplied by multiple small collaterals

the cerebrovascular reactivity of patients with MMD as the diagnostic gold standard $\mathrm{H}_{2}{ }^{15} \mathrm{O}$ PET/CT with $\mathrm{ACZ}$ challenge [11].

Abbott et al. investigated short end-expiration $\mathrm{BH}$ periods as a confounding factor of functional MRI and concluded that $\mathrm{BH}$ periods of $3 \mathrm{~s}$ could lead to focal regions of activation that might impact fMRI experiments [28]. Our investigation revealed that $\mathrm{BH}$ periods of $3 \mathrm{~s}$ are not suitable to detect global activation in the gray matter, as it is required to access the cerebrovascular reactivity of patients with MMD. The results are in accordance with

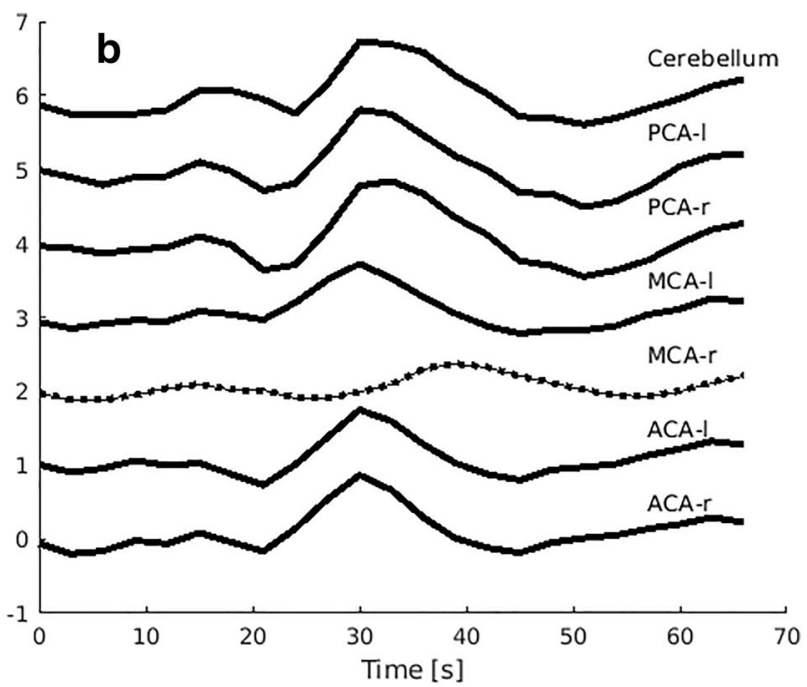

one patient with moyamoya disease (b). a Comparable signal increases in all regions indicate a symmetrical increase in perfusion in all regions during vasodilative stimulation. b Reduced signal increase is visible in the vascular territories of the right middle cerebral artery, indicating limited increase in perfusion during global dilatative stimulation 
the findings of $\mathrm{Hsu}$ et al., who detected significant BOLD signal changes in the thalamus after end-expiration $\mathrm{BH}$ periods of at least $5 \mathrm{~s}$ [20]. Hsu et al. also remarked the tendency that the gain in signal increase with the lengthening of $\mathrm{BH}$ period became smaller when the $\mathrm{BH}$ time was longer than $10 \mathrm{~s}$ [20]. End-inspiration BH tasks are not suitable for direct comparison as they are supposed to result in smaller signal increases [12]. Magon et al. detected BOLD signal values after end-inspiration $\mathrm{BH}$ periods of $15 \mathrm{~s}$ in the cortical gray matter comparable to the values after end-expiration $\mathrm{BH}$ periods of $9 \mathrm{~s}$ in this investigation [17]. According to Magon et al., endinspiration $\mathrm{BH}$ periods of $9 \mathrm{~s}$ result in too high variability of the magnitude of the response when comparing the intra-subjects' reproducibility [17]. The intra-subjects' comparison in this study revealed good reproducibility after end-expiration $\mathrm{BH}$ periods. The different BOLD signal values after end-inspiration and end-expiration $\mathrm{BH}$ periods could be explained by the larger volume of air in the lungs after inspiration that leads to a slower reduction in $\mathrm{PaO}_{2}$ (partial pressure of $\mathrm{O}_{2}$ in arterial blood) and rise in $\mathrm{PaCO}_{2}$ (partial pressure of $\mathrm{CO}_{2}$ in arterial blood) during apnea [29] than after expiration. After endinspiration $\mathrm{BH}$ periods, even an initial $\mathrm{PaCO}_{2}$ decline and $\mathrm{PaO}_{2}$ increase was described [12]. The lager gas volume after inspiration facilitates equalization between the partial pressures of $\mathrm{O}_{2}$ and $\mathrm{CO}_{2}$ in arterial blood and the gas pressures in the lungs. Conversely, the smaller lung volume after expiration causes a faster $\mathrm{CO}_{2}$ increase due to the reduced diffusion of $\mathrm{CO}_{2}$ from blood into the lungs. The higher BOLD signal increase obtained favor endexpiration over end-inspiration breath-hold periods if the $\mathrm{BH}$ periods should be as short as possible. We conducted a 3-s phase of expiration before the beginning of the $\mathrm{BH}$ periods to ensure that the $\mathrm{BH}$ periods were performed after expiration. This could be recommendable in clinical routine to ensure that the subjects stop breathing in a timely matter and that the $\mathrm{BH}$ periods are definitively performed after expiration.

\section{Paced Versus Self-Paced Breathing}

In addition to the expected signal increase, we observed a significant initial peak of smaller amplitude before the start of the expected peak in the measurements when the subjects could breathe self-paced. We attribute this additional signal change to the fact that the subjects sometimes inhaled deeply immediately before the $\mathrm{BH}$ period despite explicit instructions to refrain from doing so. Deep inspirations before the breathhold periods were proven by breath monitoring by use of a pneumatic abdominal belt. On the one hand, the reason for this may be a deliberately too deep inhalation due to the preceding breath-hold period. On the other hand, subjects could inadvertently have inhaled too deeply when the instruction "breathe in" falls into a period when they inhaled currently due to their natural breathing rhythm. An additional peak that occurs nearly at the beginning of the $\mathrm{BH}$ period and before the expected signal increase was also observed in previous endinspiration BH studies $[16,17]$. Deep inhalation directly prior to end-expiration $\mathrm{BH}$ periods as seen in our measurements might cause similar physiological reactions to endinspiration BH periods. In this study, the initial peak could be avoided by paced respiratory instructions. Due to continuous instructions, the subjects were not forewarned by the sudden command "breathe in" or interrupted in their natural rhythm and therefore carried out the last inspiration before the $\mathrm{BH}$ period in normal depth. The exact mechanism behind the signal increase after deep inhalation has not been clarified. The emergence of BOLD signal changes is supposed to be an interplay of intrathoracic pressure changes $[18,24,30]$ that could occur during deep inhalation, alterations in the delivery of oxygen [31,32], and delayed effects of variations in $\mathrm{CO}_{2}$ [19].

In consequence, paced breathing instructions seem to be useful to rectify the signal time course from unexpected signal changes. However, when comparing the expected signal increases attributable to hypercapnia, no relevant differences in the signal time courses were noticeable. The comparison of the signal time courses after paced and self-paced breathing showed neither significant differences in the amplitude of the maximum signal change, nor in the TTP, nor in the inter-trial variability. Only a slightly time-delayed signal recovery to baseline level during the paced breathing task was observed that might be explained by the limited possibility to normalize the altered $\mathrm{CO}_{2}$ concentration by means of increased respiration frequency after the breath-hold period. Therefore, we assume that the initial peak has no distinct effect on the further relevant signal time course and negligible relevance for clinical diagnostics. The examination of inter-trial variability revealed that paced breathing did not lead to a significant improvement in variability compared with self-paced breathing. Scouten et al. found out that paced breathing between end-expiration $\mathrm{BH}$ periods of $20 \mathrm{~s}$ lowers variability between trials. They explained the lower variability with the observation of a more significant post-breathhold signal undershoot present in self-paced runs. The time period of $40 \mathrm{~s}$ between two BH periods of $20 \mathrm{~s}$ was too short for signal recovery to baseline. In this investigation, $\mathrm{BH}$ periods of $9 \mathrm{~s}$ were altered with normal breathing of $60 \mathrm{~s}$ and therefore the time period was long enough for signal recovery to baseline. Moreover, we observed only rarely post-breath-hold undershoots which could be attributable to the shorter BH period. However, paced breathing instructions might be helpful to avoid BOLD signal variability in patients with very irregular breathing rhythm. 


\section{Potential Clinical Applications}

Reduced cerebrovascular reactivity can be used to assess the severity of cerebrovascular diseases and constitutes an important risk factor for ischemic stroke [33, 34]. The evaluation of CVR can serve as an indication for neurosurgical revascularization $[3,4,11,14,35-38]$, as well as the evaluation of the clinical outcome after cerebrovascular interventions [35-38].

The estimation of the cerebrovascular reactivity and the hemodynamic impairment to assess the risk of stroke is useful not only in patients with MMD but also in patients with other cerebrovascular diseases such as carotid artery stenosis [39-43]. Potential applications also include the diagnosis of gliomas $[44,45]$. Furthermore, reduced cerebrovascular reactivity is thought to be associated with a prognostic risk of dementia [46].

As opposed to nuclear medicine imaging techniques, such as $\mathrm{H}_{2}{ }^{15} \mathrm{O} \mathrm{PET} / \mathrm{CT}$, which is currently the gold standard technique in functional perfusion imaging to estimate the CVR, $\mathrm{CO}_{2}$-triggered BOLD fMRI is a widely available and less expensive method for the hemodynamic evaluation [39]. Breath-hold fMRI is an easily feasible technique that does not require the use of exogenous contrast agents or additional equipment. No doubt, the need for patient cooperation constitutes a disadvantage. To overcome this disadvantage, patient respiratory movements could be monitored by use of a respiratory belt to ensure correct execution of the breath-hold periods. Another way of verifying patient compliance is to evaluate the signal time course of the cerebellum, where distinct BOLD signal increases are expected, and to exclude individual breath-hold periods from further evaluation, in which the expected cerebellar response curve is not observed [11]. Dlamini et al. recently showed that breath-hold paradigms are well feasible even for children and lead to reliable results [47]. Therefore, breath-hold-triggered BOLD fMRI might be a promising technique for the implementation in clinical assessment.

\section{Limitations}

One limitation of our study was the young age of the subjects. The effect of age on cerebrovascular reactivity is controversially discussed $[30,48]$. In the case of an age-dependent decline in the cerebrovascular reactivity, it might be possible to detect smaller BOLD signal changes in healthy brain tissue of older patients than in the younger subjects of this study. Furthermore, partial pressures of $\mathrm{CO}_{2}$ and $\mathrm{O}_{2}$ were not monitored during data acquisition. Therefore, it could not be ensured that the detected signal changes were exclusively due to breath-hold-induced hypercapnia. Since no real-time monitoring of the $\mathrm{CO}_{2}$ pressure was performed, the BOLD signal changes could not be shown in relation to the $\mathrm{CO}_{2}$ change and only relative BOLD signal changes and a semi- quantitative analysis relative to the cerebellum could be presented. In addition, the influence of baseline $\mathrm{CO}_{2}$ levels on the further signal time course could not be investigated. In clinical routine, differing baseline $\mathrm{CO}_{2}$ levels in the lungs of patients with respiratory disorders compared with those of healthy individuals might impact the degree of $\mathrm{CO}_{2}$ increase during breath-hold and the BOLD signals [14].

\section{Conclusions}

The investigation of the influence of short end-expiration breath-hold periods on the BOLD fMRI signal revealed that a breath-hold duration of at least $9 \mathrm{~s}$ leads to distinct global BOLD signals that might be used to distinguish tissue of different cerebrovascular reactivity. Before the expected BOLD signals, we identified additional BOLD signal changes when the respiratory rhythm was not paced by the investigators. These changes can be avoided by continuously paced breathing instructions.

In conclusion, the use of end-expiration breath-hold periods of at least $9 \mathrm{~s}$ is recommended for the use of $\mathrm{CO}_{2}$-triggered fMRI technique to estimate the cerebrovascular reactivity, for example in patients with moyamoya disease. It seems to be a promising technique that is easily feasible and readily available.

Authors' Contributions Leonie Zerweck, Till-Karsten Hauser, and Uwe Klose contributed to the study conception and design. Material preparation and data collection were performed by Leonie Zerweck and Uwe Klose. Data analysis was performed by Leonie Zerweck, Till-Karsten Hauser, and Uwe Klose. The first draft of the manuscript was written by Leonie Zerweck. Till-Karsten Hauser, Constantin Roder, and Uwe Klose commented on previous versions of the manuscript. All authors read and approved the final manuscript.

Funding Information Open Access funding provided by Projekt DEAL.

\section{Compliance with Ethical Standards}

Ethical Approval All procedures performed in studies involving human participants were in accordance with the ethical standards of the institutional ethics committee and with the 1964 Helsinki declaration and its later amendments or comparable ethical standards.

Informed Consent Informed consent was obtained from all individual participants included in the study.

Conflict of Interest The authors declare that they have no conflict of interest.

Open Access This article is licensed under a Creative Commons Attribution 4.0 International License, which permits use, sharing, adaptation, distribution and reproduction in any medium or format, as long as you give appropriate credit to the original author(s) and the source, provide a link to the Creative Commons licence, and indicate if changes were made. The images or other third party material in this article are included 
in the article's Creative Commons licence, unless indicated otherwise in a credit line to the material. If material is not included in the article's Creative Commons licence and your intended use is not permitted by statutory regulation or exceeds the permitted use, you will need to obtain permission directly from the copyright holder. To view a copy of this licence, visit http://creativecommons.org/licenses/by/4.0/.

\section{References}

1. Tarasow E, Kulakowska A, Lukasiewicz A, Kapica-Topczewska K, Korneluk-Sadzynska A, Brzozowska J, et al. Moyamoya disease: diagnostic imaging. Pol J Radiol. 2011;76(1):73-9.

2. Kim JS. Moyamoya disease: epidemiology, clinical features, and diagnosis. J Stroke. 2016;18(1):2-11. https://doi.org/10.5853/jos. 2015.01627.

3. Khan N, Schuknecht B, Boltshauser E, Capone A, Buck A, Imhof HG, et al. Moyamoya disease and moyamoya syndrome: experience in Europe; choice of revascularisation procedures. Acta Neurochir. 2003;145(12):1061-71. https://doi.org/10.1007/ s00701-003-0148-5.

4. Roder C, Burkle E, Ebner FH, Tatagiba M, Ernemann U, Buck A, et al. Estimation of severity of moyamoya disease with $[(15) \mathrm{O}]$ water-positron emission tomography compared with magnetic resonance imaging and angiography. World Neurosurg. 2018;117:e75-81. https://doi.org/10.1016/j.wneu.2018.05.163.

5. Lee M, Zaharchuk G, Guzman R, Achrol A, Bell-Stephens T, Steinberg GK. Quantitative hemodynamic studies in moyamoya disease: a review. Neurosurg Focus. 2009;26(4):E5. https://doi. org/10.3171/2009.1.FOCUS08300.

6. Fierstra J, Sobczyk O, Battisti-Charbonney A, Mandell DM, Poublanc J, Crawley AP, et al. Measuring cerebrovascular reactivity: what stimulus to use? J Physiol. 2013;591(23):5809-21. https:// doi.org/10.1113/jphysiol.2013.259150.

7. Conklin J, Fierstra J, Crawley AP, Han JS, Poublanc J, Mandell DM, et al. Impaired cerebrovascular reactivity with steal phenomenon is associated with increased diffusion in white matter of patients with moyamoya disease. Stroke. 2010;41(8):1610-6. https:// doi.org/10.1161/STROKEAHA.110.579540.

8. Donahue MJ, Ayad M, Moore R, van Osch M, Singer R, Clemmons $\mathrm{P}$, et al. Relationships between hypercarbic reactivity, cerebral blood flow, and arterial circulation times in patients with moyamoya disease. J Magn Reson Imaging. 2013;38(5):1129-39. https://doi.org/10.1002/jmri.24070.

9. Han JS, Abou-Hamden A, Mandell DM, Poublanc J, Crawley AP, Fisher JA, et al. Impact of extracranial-intracranial bypass on cerebrovascular reactivity and clinical outcome in patients with symptomatic moyamoya vasculopathy. Stroke. 2011;42(11):3047-54. https://doi.org/10.1161/STROKEAHA.111.615955.

10. Mutch WA, Mandell DM, Fisher JA, Mikulis DJ, Crawley AP, Pucci O, et al. Approaches to brain stress testing: BOLD magnetic resonance imaging with computer-controlled delivery of carbon dioxide. PLoS One. 2012;7(11):e47443. https://doi.org/10.1371/ journal.pone. 0047443

11. Hauser TK, Seeger A, Bender B, Klose U, Thurow J, Ernemann U, et al. Hypercapnic BOLD MRI compared to H2(15)O PET/CT for the hemodynamic evaluation of patients with moyamoya disease. Neuroimage Clin. 2019;22:101713. https://doi.org/10.1016/j.nicl. 2019.101713.

12. Kastrup A, Li TQ, Takahashi A, Glover GH, Moseley ME. Functional magnetic resonance imaging of regional cerebral blood oxygenation changes during breath holding. Stroke. 1998;29(12): 2641-5.
13. Heyn C, Poublanc J, Crawley A, Mandell D, Han JS, Tymianski M, et al. Quantification of cerebrovascular reactivity by blood oxygen level-dependent MR imaging and correlation with conventional angiography in patients with moyamoya disease. AJNR Am J Neuroradiol. 2010;31(5):862-7. https://doi.org/10.3174/ajnr. A1922.

14. Pillai JJ, Mikulis DJ. Cerebrovascular reactivity mapping: an evolving standard for clinical functional imaging. AJNR Am J Neuroradiol. 2015;36(1):7-13. https://doi.org/10.3174/ajnr.A3941.

15. Kastrup A, Kruger G, Neumann-Haefelin T, Moseley ME. Assessment of cerebrovascular reactivity with functional magnetic resonance imaging: comparison of $\mathrm{CO}(2)$ and breath holding. Magn Reson Imaging. 2001;19(1):13-20.

16. Herrera CR, Beltramini GC, Avelar WM, Lima FO, Li LM. Cerebral vasomotor reactivity assessment using transcranial Doppler and MRI with apnea test. Braz J Med Biol Res. 2016;49(11):e5437. https://doi.org/10.1590/1414-431X20165437.

17. Magon S, Basso G, Farace P, Ricciardi GK, Beltramello A, Sbarbati A. Reproducibility of BOLD signal change induced by breath holding. Neuroimage. 2009;45(3):702-12. https://doi.org/ 10.1016/j.neuroimage.2008.12.059.

18. Nakada K, Yoshida D, Fukumoto M, Yoshida S. Chronological analysis of physiological T2* signal change in the cerebrum during breath holding. J Magn Reson Imaging. 2001;13(3):344-51.

19. Birn RM, Smith MA, Jones TB, Bandettini PA. The respiration response function: the temporal dynamics of fMRI signal fluctuations related to changes in respiration. Neuroimage. 2008;40(2): 644-54. https://doi.org/10.1016/j.neuroimage.2007.11.059.

20. Hsu YY, Kuan WC, Lim KE, Liu HL. Breathhold-regulated blood oxygenation level-dependent (BOLD) MRI of human brain at 3 tesla. J Magn Reson Imaging. 2010;31(1):78-84. https://doi.org/ 10.1002/jmri.22015.

21. Kastrup A, Kruger G, Glover GH, Moseley ME. Assessment of cerebral oxidative metabolism with breath holding and fMRI. Magn Reson Med. 1999;42(3):608-11.

22. Liu HL, Huang J, Wu CT, Hsu YY. Detectability of blood oxygenation level-dependent signal changes during short breath hold duration. Magn Reson Imaging. 2002;20(9):643-8.

23. Scouten A, Schwarzbauer C. Paced respiration with end-expiration technique offers superior BOLD signal repeatability for breath-hold studies. Neuroimage. 2008;43(2):250-7. https://doi.org/10.1016/j. neuroimage.2008.03.052.

24. Thomason ME, Glover GH. Controlled inspiration depth reduces variance in breath-holding-induced BOLD signal. Neuroimage. 2008;39(1):206-14. https://doi.org/10.1016/j.neuroimage.2007.08. 014.

25. Mutsaerts HJ, van Dalen JW, Heijtel DF, Groot PF, Majoie CB, Petersen ET, et al. Cerebral perfusion measurements in elderly with hypertension using arterial spin labeling. PLoS One. 2015;10(8): e0133717. https://doi.org/10.1371/journal.pone.0133717.

26. Zerweck L, Hauser TK, Klose U. The influence of short breath-hold periods on blood-oxygen-level-dependent (BOLD) MRI signal. Book of Abstracts: ESMRMB 2019, 36th Annual Scientific Meeting, Rotterdam, NL, October 3-October 5. MAGMA. 2019;32(Suppl 1):333-4. https://doi.org/10.1007/s10334-01900755-1.

27. Zerweck L, Hauser TK, Roder C, Klose U. Untersuchung des fMRT-BOLD-Signalverlaufs während kurzer Atempausen zur Beurteilung der zerebrovaskulären Reaktivität. Clin Neuroradiol. 2019;29(1):S42. https://doi.org/10.1007/s00062-019-00826-9.

28. Abbott DF, Opdam HI, Briellmann RS, Jackson GD. Brief breath holding may confound functional magnetic resonance imaging studies. Hum Brain Mapp. 2005;24(4):284-90. https://doi.org/10. 1002/hbm.20086

29. Overgaard K, Friis S, Pedersen RB, Lykkeboe G. Influence of lung volume, glossopharyngeal inhalation and $\mathrm{P}(\mathrm{ET}) \mathrm{O} 2$ and $\mathrm{P}(\mathrm{ET}) \mathrm{CO} 2$ 
on apnea performance in trained breath-hold divers. Eur J Appl Physiol. 2006;97(2):158-64. https://doi.org/10.1007/s00421-0060156-2.

30. Thomason ME, Burrows BE, Gabrieli JD, Glover GH. Breath holding reveals differences in $\mathrm{AMRI}$ BOLD signal in children and adults. Neuroimage. 2005;25(3):824-37. https://doi.org/10.1016/j. neuroimage.2004.12.026.

31. Bulte D, Chiarelli P, Wise R, Jezzard P. Measurement of cerebral blood volume in humans using hyperoxic MRI contrast. J Magn Reson Imaging. 2007;26(4):894-9. https://doi.org/10.1002/jmri. 21096.

32. Bulte DP, Chiarelli PA, Wise RG, Jezzard P. Cerebral perfusion response to hyperoxia. J Cereb Blood Flow Metab. 2007;27(1):6975. https://doi.org/10.1038/sj.jcbfm.9600319.

33. Molina C, Sabin JA, Montaner J, Rovira A, Abilleira S, Codina A. Impaired cerebrovascular reactivity as a risk marker for first-ever lacunar infarction: A case-control study. Stroke. 1999;30(11): 2296-301. https://doi.org/10.1161/01.str.30.11.2296.

34. Liu M, Zhou L. Cerebrovascular reserve may be a more accurate predictor of stroke than degree of ICA or MCA stenosis. Med Sci Monit. 2014;20:2082-7. https://doi.org/10.12659/MSM.892377.

35. Mikulis DJ, Krolczyk G, Desal H, Logan W, Deveber G, Dirks P, et al. Preoperative and postoperative mapping of cerebrovascular reactivity in moyamoya disease by using blood oxygen leveldependent magnetic resonance imaging. J Neurosurg. 2005;103(2):347-55. https://doi.org/10.3171/jns.2005.103.2.0347.

36. Cook DJ, Mukerji N, Furtado SV, Steinberg GK. Moyamoya Disease. PanVascular Medicine. 2015:2943-70. https://doi.org/ 10.1007/978-3-642-37078-6 102.

37. Goetti R, Warnock G, Kuhn FP, Guggenberger R, O'Gorman R, Buck A, et al. Quantitative cerebral perfusion imaging in children and young adults with moyamoya disease: comparison of arterial spin-labeling-MRI and $\mathrm{H}(2)[(15) \mathrm{O}]$-PET. AJNR Am J Neuroradiol. 2014;35(5):1022-8. https://doi.org/10.3174/ajnr. A3799.

38. Mandell DM, Han JS, Poublanc J, Crawley AP, Fierstra J, Tymianski M, et al. Quantitative measurement of cerebrovascular reactivity by blood oxygen level-dependent MR imaging in patients with intracranial stenosis: preoperative cerebrovascular reactivity predicts the effect of extracranial-intracranial bypass surgery. AJNR Am J Neuroradiol. 2011;32(4):721-7. https://doi.org/10. 3174/ajnr.A2365.

39. Fierstra J, van Niftrik C, Warnock G, Wegener S, Piccirelli M, Pangalu A, et al. Staging hemodynamic failure with blood oxygen-level-dependent functional magnetic resonance imaging cerebrovascular reactivity: a comparison versus gold standard ((15)O-)H2O-positron emission tomography. Stroke. 2018;49(3): 621-9. https://doi.org/10.1161/STROKEAHA.117.020010.
40. Markus H, Cullinane M. Severely impaired cerebrovascular reactivity predicts stroke and TIA risk in patients with carotid artery stenosis and occlusion. Brain. 2001;124(Pt 3):457-67. https://doi. org/10.1093/brain/124.3.457.

41. Schaaf M, Mommertz G, Ludolph A, Geibprasert S, Muhlenbruch $\mathrm{G}$, Das M, et al. Functional MR imaging in patients with carotid artery stenosis before and after revascularization. AJNR Am J Neuroradiol. 2010;31(10):1791-8. https://doi.org/10.3174/ajnr. A2219.

42. Gottler J, Kaczmarz S, Kallmayer M, Wustrow I, Eckstein HH, Zimmer $\mathrm{C}$, et al. Flow-metabolism uncoupling in patients with asymptomatic unilateral carotid artery stenosis assessed by multimodal magnetic resonance imaging. J Cereb Blood Flow Metab. 2018:271678X18783369. https://doi.org/10.1177/ $0271678 X 18783369$.

43. Kaczmarz S, Petr J, Sollmann N, Hock A, Zimmer C, Hyder F, et al. Recovery of cerebrovascular reactivity after treatment of asymptomatic carotid artery stenosis is assessable by non-invasive breath-hold fmri within global watershed areas. Clin Neuroradiol. 2019;29(1):S86. https://doi.org/10.1007/s00062-019-00826-9.

44. Fierstra J, van Niftrik C, Piccirelli M, Bozinov O, Pangalu A, Krayenbuhl N, et al. Diffuse gliomas exhibit whole brain impaired cerebrovascular reactivity. Magn Reson Imaging. 2018;45:78-83. https://doi.org/10.1016/j.mri.2017.09.017.

45. Iranmahboob A, Peck KK, Brennan NP, Karimi S, Fisicaro R, Hou $\mathrm{B}$, et al. Vascular reactivity maps in patients with gliomas using breath-holding BOLD fMRI. J Neuroimaging. 2016;26(2):232-9. https://doi.org/10.1111/jon.12278.

46. Wolters FJ, de Bruijn RF, Hofman A, Koudstaal PJ, Ikram MA. Heart brain connection collaborative research G. cerebral vasoreactivity, apolipoprotein $\mathrm{E}$, and the risk of dementia: a population-based study. Arterioscler Thromb Vasc Biol. 2016;36(1):204-10. https://doi.org/10.1161/ATVBAHA.115. 306768 .

47. Dlamini N, Shah-Basak P, Leung J, Kirkham F, Shroff M, Kassner A, et al. Breath-hold blood oxygen level-dependent MRI: a tool for the assessment of cerebrovascular reserve in children with moyamoya disease. AJNR Am J Neuroradiol. 2018;39(9):171723. https://doi.org/10.3174/ajnr.A5739.

48. Kastrup A, Dichgans J, Niemeier M, Schabet M. Changes of cerebrovascular $\mathrm{CO} 2$ reactivity during normal aging. Stroke. 1998;29(7):1311-4.

Publisher's Note Springer Nature remains neutral with regard to jurisdictional claims in published maps and institutional affiliations. 